\title{
Erratum: MHC class I and MHC class II DRB gene variability in wild and captive Bengal tigers (Panthera tigris tigris)
}

Ina Pokorny • Reeta Sharma •

Surendra Prakash Goyal - Sudanshu Mishra •

Ralph Tiedemann

Published online: 14 December 2010

(C) Springer-Verlag 2010

Erratum to: Immunogenetics (2010) 10 667-679

Pati-MHCI*0202 HQ157997

DOI 10.1007/s00251-010-0475-7

The accession numbers presented in the results section of that paper are unfortunately incorrect. The correct gene bank accession numbers are as follows:

Pati-MHCI*0101 HQ157994

Pati-MHCI*0102 HQ157995

Pati-MHCI*0201 HQ157996

Pati-MHCI*0301 HQ157998

Pati-MHCI*0401 HQ157999

Pati-MHCI*0501 HQ158000

Pati-MHCI*0601 HQ158001

Pati-MHCI*0701 HQ158002

Pati-MHCI*0702 HQ158003

Pati-MHCI*0801 HQ158004

Pati-MHCI*0901 HQ158005

Pati-MHCI*0902 HQ158006

Pati-MHCI*PS01 HQ158007

The online version of the original article can be found at http://dx.doi. org/10.1007/s00251-010-0475-7.

I. Pokorny $\cdot$ R. Tiedemann $(\bowtie)$

Unit of Evolutionary Biology/Systematic Zoology,

Institute of Biochemistry and Biology, University of Potsdam,

Karl-Liebknecht-Str. 24-25, Haus 26,

14476, Potsdam, Germany

e-mail: tiedeman@uni-potsdam.de

R. Sharma - S. P. Goyal - S. Mishra

Wildlife Institute of India,

P.O. Box 18, Chandrabani,

Dehradun 248001 Uttarakhand, India

R. Sharma

Instituto Gulbenkian de Ciência,

Rua da Quinta Grande 6,

2780-156, Oeiras, Portugal 